\title{
Les phares antiques, entre défense et aide à la navigation. Exemples en Méditerranée Occidentale
}

Jonatan Christiansen

Université Lyon 2 - HiSoMA UMR 5189, Lyon, France, jchristiansen@free.fr

\begin{abstract}
Beyond the great Hellenistic tower of Alexandria, archaeological studies have revealed an important number of ancient lighthouses through all the Mediterranean Sea since more than fifty years. The consideration of several coastal towers and new discoveries provide data to undertake new issues on this diachronic topic.

The study of their architecture can now be completed by the analysis of their implantation in ports, along coasts or on islands. The strategy of their location has to provide secure information for the mariners in day and night time conditions and should be different from other coastal constructions. The study of their specific maritime roles is still an untapped area of research, especially from the sea, from the mariners' point of view.

However, ancient lighthouses are not always exclusive signalling devices for maritime trade. As for later period, coastal towers could ensure several tasks, including surveillance, control and communication. The division between a civilian and military device is not always clearly defined.

The study of ancient lighthouses is also a way to deal with structures, which has been used or reused during later period. Indeed, there are clear correlations with later signalling devices as contemporaneous lighthouses built on the same spot of ancient ones. Furthermore, some of them were transformed into mills, granaries or habitations when others have been fortified to address defensive concerns during the modern period.
\end{abstract}

Keywords: Coastal archaeology, Lighthouses, Ancient harbors, Defensive system.

\section{Introduction}

La signalisation maritime est une forme particulière d'aménagement des littoraux. Le développement de constructions spécifiques correspond à une pratique attestée depuis le 6ème siècle avant J.-C. dans le monde grec puis dans toute la méditerranée durant l'Antiquité. Les données existantes et les nouvelles découvertes alimentent un corpus hétérogène qui se caractérise par la grande diversité des types d'aménagement. La recherche et la compilation de ces vestiges doivent permettre de classer et hiérarchiser les dispositifs. Leur rôle maritime ne peut être étudié et défini que par l'étude des caractéristiques architecturales et en fonction de leur contexte d'implantation.

Cette diversité incite à engager une réflexion sur la notion même de signalisation maritime dans l'Antiquité. Si les sources iconographiques permettent de reconnaître un type de bâtiment original, la tour à degrés, l'étude des sources littéraires démontre l'absence d'un vocabulaire spécifique et la signalisation maritime n'est pas toujours dissociée des activités (le plus souvent militaires) de surveillance, d'observation et de contrôle des espaces en mer. 
A travers une sélection de vestiges archéologiques en Méditerranée occidentale, il est possible d'appréhender les phares et les tours côtières isolées sur lesquels s'appuient la signalisation maritime antique.

\section{Les phares romains en Méditerranée Occidentale}

Les aides à la navigation que sont les tours de signalisation maritime répondent à des besoins spécifiques liés à la nature des côtes et des environnements. Par exemple, un cap qui se détache le long d'une côte ne sera pas visible en arrivant de la haute mer puisque la perspective écrase le relief sur un même plan (Arnaud 2005, 30-34). L'implantation d'une tour permettra de cristalliser cette information et de fournir un repère visuel (amer) qui peut fonctionner en condition diurne ou nocturne. Les tours n'offrent pas les mêmes indications aux marins qui naviguent depuis la haute mer ou sur un itinéraire de cabotage.

Une première distinction existe entre des dispositifs qui visent à sécuriser les abords d'un port et ceux qui marquent un environnement particulier comme des îles ou par exemple, le passage d'un détroit. Les tours de Sestos et Abydos, dans l'Hellespont, représentées sur des monnaies romaines (sous règnes de Septime Sévère et Caracalla), sont évocatrices. Si elles ne sont pas des 'phares', leur utilisation par les marins est indéniable, dans un environnement où il est difficile de manœuvrer. Strabon décrit avec précision une manœuvre de virement de bord nécessaire dans un détroit où la traversée en ligne directe n'est pas aisée (Strabon, Geo. 13.1 22). Les tours constituent des points fixes utiles à cette manœuvre.

En méditerranée occidentale, on peut évoquer le détroit de Messine réputé pour sa dangerosité, que rappelle la légende des monstres marins Charybde et Scylla. D'une importance stratégique pour le contrôle et la sécurisation des échanges maritimes, une tour de signalisation dans ce lieu est une aide précieuse. Au cap Peloro, la découverte puis la fouille entre 2003 et 2006 d'un podium monumental de 25 mètres de côté, sous la tour de Anglais, qui a été interprété comme les vestiges d'une tour de signalisation, un phare, érigé au Ier siècle avant J.-C. (Tigano 2011). Les dimensions de la plateforme sont très proches de celle des fondations de la Torre Hercules à La Corogne ou du phare impérial de Leptis Magna. La tour devait posséder une élévation entre 30 et 40 mètres ce qui lui permet de posséder un rayon théorique de visibilité d'environ $25 \mathrm{~km}$. Comme pour le détroit de l'Hellespont, la tour du cap Peloro joue un rôle majeur dans la traversée ou le franchissement.

Les phares «insulaires» sont aussi des dispositifs particuliers liés à la nature des terres balisées. Ainsi, les tours octogonales bâties à Giannutri sur le Monte della Scoperta et à Giglio sur le site de Castellari (Bronson, Uggeri 1970; Rendini 2009) ${ }^{1}$ sont interprétées comme des dispositifs de signalisation maritime. Elles sont datées de la fin du 1er ou du 2è siècle après J.-C. A Giannutri, la tour est érigée à 78 mètres au dessus du niveau de la mer. Cette situation topographique lui confère un rayon théorique de visibilité d'environ $30 \mathrm{~km}$. Cette implantation offre une vue panoramique sur les abords nord, est et sud de l'île. La tour communique visuellement avec le port de Cala Mestra qui lui ne peut voir qu'en direction de l'ouest.

Il existe au moins un autre exemple de tour de signalisation sur une île de Méditerranée occidentale attestée par des vestiges ${ }^{2}$, à Capri. Elle s'apparente en tout point à un phare mais le contexte de construction et son utilisation sont liés au complexe impériale de la Villa Jovis (Maiuri 1956, 24). Si cette tour correspond à celle évoquée par Suétone lors de la mort de Tibère (Vie de Tibère, 74), il s'agit d'un dispositif de signal qui n'appartient qu'à l'Empereur et n'est pas spécifiquement dédié à l'assistance des navires. Son implantation à presque 300 mètres au dessus du niveau de la mer offrait une vue stratégique sur toute la zone en direction du sud et permettait de prévenir l'arrivée de navires dans la baie de Naples. Cette tour ne pouvait en revanche pas communiquer avec Misène car la Villa bouchait la vue en direction du nord (Krause 2005, 48, 251). Elle est en communication visuelle avec un autre 
dispositif de signalisation sur la pointe du cap de Punta della Campanella qui constitue un relai qui peut émettre et recevoir des signaux en direction du cap Misène (Giardina 2010, 96, 292).

Les tours érigées dans les ports ou à proximité immédiate jouent un rôle différent. Elles matérialisent un lieu sûr ou des dangers et permettent au pilote d'emprunter une voie saine. Les tours monumentales comme celle d'Alexandrie peuvent cumuler plusieurs fonctions. Dans ce cas, elle matérialise des terres basses invisibles lors des phases d'atterrage et indique les dangers de la passe d'entrée mais pas l'entrée du port. Sa position sert de point d'appui pour des alignements qui permettent d'éviter les récifs.

L'aménagement de plusieurs ports romains semblent démontré une influence du paysage et la configuration du port d'Alexandrie. Les ports de Césarée de Maurétanie en Algérie, de Centumcellae et Portus en Italie sont des exemples particulièrement évocateurs. Dans le premier cas, un phare a été bâti au 1er siècle avant J.-C. sur l'îlot de Joinville. Les premières assises d'une tour octogonale de $18 \mathrm{~m}$ de large ont été mises au jour à la fin des années 1950 (Lassus 1958). Si les vestiges ne permettent pas d'établir avec certitude l'architecture de la construction, une tour à degrés est restituée. La configuration de l'espace portuaire est très proche du paysage alexandrin, à moindre échelle. Le phare indique la présence de l'île protège le port et constitue un point d'alignements avec d'autres constructions (Janon 1991, 753-755).

À Centumcellae et Portus, la construction d'une île artificielle au centre de l'entrée du port, entre les deux moles, semble également faire écho au paysage alexandrin sans toute fois le reproduire exactement (Quilici 2004; Morelli et al. 2001). Ces îlots artificiels qui portent des tours de signalisation sont des points d'ancrage pour la phase d'entrée au port et des points d'alignements avec d'autres édifices du port (et d'autres tours comme celle encore visible sur le mole lazzaretto à Centumcellae ou sur le mole qui borde l'accès à la darse et à l'octogone de
Trajan sur le site de Portus). On peut également s'interroger sur le rôle de ces tours dans la circulation des navires dans le port. Est-ce qu'un sens giratoire autour de ces phares a pu être instauré?

Un autre modèle, plus facile à mettre en œuvre, place la tour de signalisation, le phare ou une balise à l'extrémité d'une jetée. Dans ce cas, le dispositif offre une forme de balisage de l'entrée en indiquant précisément les contours des infrastructures portuaires. C'est le cas dans le port romain de Thapsus en Tunisie (Younes 1999, 190-191) et probablement dans celui du de Sagunte, Grau Vell, en Espagne (De Juan Fuertes 2002, 2003). Les deux sites sont aujourd'hui submergés.

La nature de la côte est le facteur déterminant de la fonction et du rôle de ces aménagements spécifiques qui ont pour but de répondre à des besoins maritimes. Tous ces exemples ne présentent pas de caractéristiques architecturales défensives et il ne s'agit pas de fortifications maritimes. Cependant, toutes ces tours que l'on considère comme des phares et qui sont mises en parallèles avec des dispositifs modernes possèdent une dimension stratégique supplémentaire qui découle directement du fonctionnement de l'activité de signalisation.

A la différence des pratiques contemporaines où les feux sont allumés en permanence, les phares et les tours de signalisation antiques sont dépendants d'une activité complémentaire d'observation et de guet. Il faut considérer que la signalisation maritime antique n'est pas une activité permanente, il semble que ce soit le passage de navires au large ou des conditions climatiques qui conditionnent le fonctionnement des dispositifs. L'absence d'un vocabulaire spécifique et entièrement dédié à ces constructions est un indice tangible en faveur de cette hypothèse.

\section{La notion de doublon}

L'épigramme du poète Poseiddippos de Pella (Épigramme 115, P. Louvre 7172) sur le phare d'Alexandrie décrit précisément une tour qui cumule les fonctions de surveillance et de signalisation. C'est la position stratégique de la 
tour qui lui confère cette double capacité. L'absence de hauteurs, de points remarquables sur la côte égyptienne et en particulier dans le delta est la cause principale de la construction d'une tour monumentale, qui devient une hauteur artificielle. La construction est à la fois, le lieu haut perché de la côte à partir duquel les gens du littoral observent mais aussi un point de repère, un amer pour les marins ${ }^{3}$. Cette construction leur permet de situer l'approche des terres avant qu'elles ne soient effectivement visibles. Le passage de Strabon sur les pyrgoi des massaliotes dans le delta du Rhône exprime très exactement les mêmes besoins et le même but à atteindre (Geo, 4. 1.8): fournir un signe aux marins qui matérialise la présence des côtes.

L'interaction avec la mer qui est au cœur de l'action de signaler repose sur une stratégie d'implantation précise. La position choisie permet un équilibre entre voir et être vu. Or la notion de guet est toujours associée à celle d'émettre un signal, une position ou des informations supplémentaires. Le guetteur est associé avec la communication - car voir sans pouvoir transmettre n'a plus de pertinence. Sa tache consiste à voir puis à transmettre, soit en direction des terres, soit à destination de la mer ${ }^{4}$.

Les speculae sont des lieux de guet mais aussi des lieux d'où l'on transmet des signaux. Il y a une réelle continuité avec le monde grec où les notions de Skopeion et de Phryktoria sont étroitement liées. L'épisode le plus souvent invoqué pour illustrer cette relation est l'annonce de la chute de Troie. Les textes d'Homère et d'Eschyle montrent à quel point ce sont des éléments indissociables. Le terme Phryktorion désigne la transmission de signaux et Phryktos les torches utilisées.

La majorité des occurrences concerne l'usage de cette pratique dans un contexte de guerre et de prévention d'attaques terrestres ou maritimes, pourtant de nombreux auteurs emploient les mêmes termes pour décrire des dispositifs signalisation maritime. Ainsi, Hérodien évoque les phares dans leur ensemble, comme des

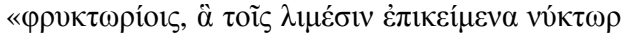

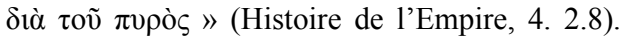
De même, Dion Cassius décrit le phare de
Claude à Portus comme une tour qui est aussi un

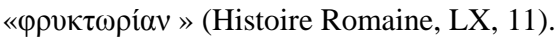

Même à partir de la diffusion tardive du mot Pharos, les mots Pyrgos et Phryktorion continuent d'être employé pour désigner des phares (Quet 1984). Il s'agit toujours de tours, qui se distinguent par leur capacité à porter des feux en direction de la mer. Si toutes les tours côtières ne sont pas des phares, tous les phares sont des tours côtières. Cette définition permet d'englober d'autres sites qui correspondent à ce type d'aménagement.

Trois exemples permettent d'illustrer ce propos:

- La tour romaine (2è-4è siècle après J.-C.) de l'isoletto di Bergeggi dans le golf Ligure (Bulagrelli 2003, 215-217). Située sur le point haut de l'île (+30 m au dessus du niveau de la mer), cette construction circulaire érigée sur une plateforme triangulaire peut assurer la fonction d'un phare. La restitution d'une rampe hélicoïdale qui permettrait le transport de combustible sur la partie sommitale semble confirmer cette fonction, ce qui n'exclut pas la dimension stratégique de communication avec le port de Vada. Le rôle de cette tour peut aussi être de prévenir la cité de l'arrivée de navires et mettre en place une surveillance.

- La tour romaine (1er siècle après J.-C.) du l'îlot du Lion de Mer en face de Fréjus présente une configuration très similaire même si l'état de conservation des vestiges ne permet pas de restituer avec certitude son architecture (Gébara, Morhange 2010, 77). Située sur le sommet de l'îlot à environ 25 mètres au dessus du niveau de la mer, la tour peut communiquer avec le port militaire et signaler un itinéraire sain qui longe la côte à partir de l'espace entre le Lion de mer et le Lion de terre, jusqu'à Fréjus. L'ouest de l'embouchure de l'Argens est aujourd'hui caractérisé par des obstructions et la dangerosité des eaux. On peut s'interroger, malgré la grande mobilité du littoral depuis cette période, si l'une des motivations de l'implantation de cette tour ne répond pas à des préoccupations liées à ces données bathymétriques.

- Le site Roccia del Picchione, situé entre le promontoire de Torre truglia et la villa maritime 
de Sperlonga en Italie (Quilici 2009, 255-257). Le pic rocheux au sommet duquel a été aménagé une construction interprétée comme une station de signal ou un specolae se situe à $420 \mathrm{~m}$ du rivage actuel. Le site est à plus de 100 mètres d'altitude. Ce dispositif complète série de points remarquables sur la portion littorale entre le Mont Circei et le promontoire de Gaete. Si il ne s'agit pas d'un phare, une tour à feu peut assurer des communications rapides mais aussi une tache de signalisation maritime en matérialisant la côte.

Les dispositifs de signalisation sont des marqueurs du paysage littoral qui contribuent au développement de l'identité d'un espace maritime. Au delà de l'aide à la navigation, la tour côtière est aussi un lieu d'observation stratégique qui permet d'assurer des tâches de surveillance et de contrôle sur une frontière naturelle qui est le point faible des territoires, ou la portion la moins bien maîtrisée : le littoral. Cela n'exclut en rien une vocation de signalisation et la capacité de l'exécuter.

Le fonctionnement de ces dispositifs doit s'appuyer plus logiquement sur l'emploi de torches et de signaux plutôt que sur l'entretien de foyers ouverts ce qui n'est pas forcément synonyme d'une efficacité moindre.

\section{Corrélation avec des aménagements postérieurs}

L'importance et l'utilité stratégique des sites évoqués s'illustrent tout particulièrement lorsque les emplacements sont réutilisés au cours d'époques postérieurs. Dans certains cas, la construction antique est réaménagée, restaurée ou modifiée pour assurer des taches défensives et/ ou maritimes. Ainsi, les tours du port de Centumcellae ont été fortifiées à la Renaissance et aménagées pour recevoir des canons. La fonction de phare de la tour demeure. La tour de
Bergeggi a été englobée par une tour médiévale quadrangulaire de plus grandes dimensions qui a assuré des taches de surveillance du littoral.

Des phares modernes ou des balises de signalisation ont été érigés sur les sites de Cherchell, du cap Peloro ou de l'îlot du Lion de Mer. Ces dispositifs fonctionnent encore aujourd'hui pour la sécurité des marins. La permanence stratégique de ces emplacements démontre d'une part la dimension diachronique $\mathrm{du}$ sujet et d'autre part la formidable valeur patrimoniale de ces constructions.

\section{Notes}

(1) Et peut-être à Ventotene (De Rossi 1993, 54)

(2) Une construction quadrangulaire datée du $2^{\mathrm{e}}$ siècle après J.-C. est interprétée comme les vestiges d'un probable phare à Bou Son sur l'île de Menorca (Ramis i Ramis, de Palol Salellas 1988, 21-24 ; Tabvla Imperii Romani: Hoja K/J31)

(3) Xavier Corré s'est interrogé sur cette notion de doublon et la possibilité que l'observation soit complétée par une assistance aux navigateurs en difficulté, que l'on repère depuis la tour. (2004).

(4) Les hauteurs à partir desquelles sont observés les mouvements des navires (en contexte de guerre ou de paix) sont destinées à transmettre l'information à la cité. De même, les guettes à thons servent pour observer les passages des bancs et à les signaler aux pêcheurs sur leurs embarcations. Là encore, l'observation et le fait de transmettre une information sont

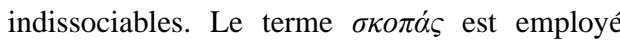
pour désigner des guettes pour la pêche, comme celle décrites dans un calendrier des sacrifices à Cos au I ${ }^{\mathrm{er}}$ av. J.-C. Strabon emploi également ce terme pour décrire les tours à thon (5. 2.6 et 17 . 3.16).

\section{References}

Arnaud P. (2005). Les routes de la navigation Antique. Errance. p. 248.

Bronson R. L., Uggeri G. (1970). "Isola del Giglio, Isola di Giannutri, Monte Argentario, Laguna di Orbetello (Notizia preliminare dei rinvenimenti del 1968)", Studi Etruschi 38, pp. 201-214. 
Bulagrelli F. (2003). Ritrovamenti di età romana nell'isola di Bergeggi: alcune reflessioni. In Pasqualini et al. (2003). Des îles côte à côte. Histoire du peuplement des îles de l'Antiquité au Moyen-Âge (Provence, Alpes-Maritimes, Ligurie, Toscane). pp. 211-220.

Corré X. (2004). Des dispositifs pour matérialiser les littoraux maritimes dans l'Antiquité et au MoyenÂge. Gallina Zevi A., Turchetti R. ANSER Le Strutture dei porti e degli approdi antichi. II Seminario. Roma-Ostia Antica,16-17 aprile 2004. p. 310.

De Juan Fuertes, C. (2002). "Primera aproximación a la infraestructura portuaria saguntina". Saguntum: Papeles del Laboratorio de Arqueología de Valencia 34. pp. 115-126

De Juan Fuertes C. (2003). "La arqueología subacuática saguntina y el Grau Vell. Estado de la cuestión". Saguntum: Papeles del Laboratorio de Arqueología de Valencia 35. pp. 229-235

De Rossi G. M. (1993). Ventotene e Santo Stefano: un'agile ma esauriente guida per la riscoperta storica, archeologica e naturalistica delle due isole e per una loro "rilettura" nel Museo di Ventotene. Guido Guidotti. p. 104.

Donati L., Cappuccini L. (2009). "Postazioni per segnali luminosi ( $\varphi \omega ́ \tau \alpha \pi v \rho \sigma \omega ́ v)$ a Punta Ala", in Uggeri G., Manrangio C., Laudizi G. (Eds), Palaia philia: studi di topografia antica in onore di Giovanni Uggeri. Journal of ancient topography. Supplemento 4, pp. 381-388.

Gébara C., Morhange C. (2011). Fréjus (Forum Iulii) : Le port Antique, JRA, Supplementary series ${ }^{\circ}$ 77. Portsmouth. p. 152.

Giardina B. (2010). Navigare Necesse est: Lighthouses, from Antiquity to Middle ages. BAR International Series 2096. p. 348.

Janon M. (1991). De Judée en Narbonnaise, reconnaissance de quelques sanctuaire du pouvoir. MEFRA. 103-2. pp. 735-783.

Krause C. (2005). Villa Jovis: l'edificio residenziale, Napoli, p. 319.

Lassus J. (1959). "Les récentes découvertes à Cherchel". Comptes-rendus des séances de l'Académie des Inscriptions et Belles-Lettres. pp. 215-225.

Maiuri A. (1956). Capri: storia e monumento. Itinerari dei musei, gallerie, scavi e monumenti d'Italia Istituto poligrafico e Zecca dello Stato, Libreria dello Stato. P. 135.

Morelli C., Marinucci A., Arnoldus-Huyzendveld A. (2011). "Il porto di Claudio: Nuove Scoperte". $47-$ 66. In Keay S., Paroli L. Portus and its hinterland: recent archaeological research. British School at Rome. pp. 319.

Quilici L. (2004). "Per il restauro del faro del Lazzaretto a Civitavecchia" in Giacobelli 2004. Lezioni Fabio Faccenna: conferenze di archeologia subacquea (III-V ciclo). pp. 111-118.

Quilici L. (2009). Praetorium Speluncae. Ricerche sui confini della proprietà imperiale. ATTA (Atlante tematico di topografia antica). 19. pp. 201-321.

Quet M.-H. (1984). Pharus, MEFRA, pp. 96-2, 789-845

Rendini R. (2009)." I fari antichi di Giglio e Giannutri, Un aggiornamento" in Uggeri G., Manrangio C., Laudizi G. (Eds). Palaia philia: studi di topografia antica in onore di Giovanni Uggeri. Journal of ancient topography. Supplemento 4. pp. 389-396.

Ruotolo A. (2013) "Il fortino S. Pietro e il molo del Lazzaretto nel Porto di Traiano". Forma Urbis VII Premio. pp. 28-38.

Tabvla Imperii Romani: Hoja K/J-31: Pyrénées orientales - Baleares - Tarraco - Baliares. Sobre la base cartográfica del mapa a escala 1:1.000.000 del IGN. (1997). Unión académica internacional. Comité español. Centro Nacional de Información Geográfica. Madrid.

Tigano G. (2011). Messina: scavi a Ganzirri e a Capo Peloro (2003-2006). Soprintendenza beni culturali e ambientali di Messina. Unità operativa X, p. 190.

Younes A. (1999). L'installation portuaire de Thapsus: mise au point à partir des textes anciens et de la documentation archéologique. Cahiers du C.E.R.E.S Série géographique n² 29. .pp. 181-193. 